\title{
Zeno, The Mobile Controlled Cognizant Biped
}

\author{
Thejaswini S, Sowmyashree M S, Saritha I G, Surekha R Gondkar \\ Department of Telecommunication Engineering, BMSIT $T^{1,2,3,4}$ \\ Assistant prof ${ }^{1,2,3}$, Associate prof ${ }^{4}$.
}

\begin{abstract}
An important research area is Bipedal Robots and their exists several types of robots like WABIAN2R, QRIO, ASIMO, REEM-B, ToyotaWalking, Toyota iFoot and HPR-2. There is a rapid increase in building a bipedal and humanoid robot during the last few years. In this paper, we provide a brief study of ZENO, the Bipedal Robot, whose activities are controlled by a mobile application on Android Platform. This paper presents the first steps toward generating robotic walking from human walking data through the use of human-inspired control. In this paper, we propose a motion planning method of a biped robot. Robots need sensors to understand circumstances. This mobile controlled application allows for a flexible use of the bipedal robot wherein applications that do not permit entry of humans can be accomplished by the use of bipedal robots.
\end{abstract}

Index Terms: - Bipedal Robots, Android Platform, Zeno

\section{INTRODUCTION}

Biped robots are 2-legged robots which can imitate human gait. Biped robots represent a very interesting research subject. There are several particularities and scope topics, like mechanical design, gait simulation, patterns generation, kinematics, dynamics, equilibrium, stability, and different kinds of control, adaptability, biomechanics, cybernetics, and rehabilitation technologies. There are diverse problems related to these topics. The study of biped robots is a very complex subject, and many times there is no total satisfaction in results of researches. Many researchers with theoretical and experimental works have collaborated in evolution of biped robotic design using advancement in science and technology. They developed biped robots to develop autonomous systems and to help in rehabilitation technologies of human beings.

To build and study humanoid robots, the human structure and behavior has to be understood by the researcher. Human cognition is a field which focuses on how humans learn from sensory information in order to acquire perceptual and motor skills. This knowledge is used in developing computational models of human behavior and it has been improving over time. It has been suggested that very advanced robotics will facilitate the enhancement of ordinary humans. The aim of the humanoid research was to build better orthosis and prosthesis for human beings. They are developed to perform human tasks like personal assistance for sick, elder and dangerous jobs. Some of the examples of humanoid robots are powered leg prosthesis, ankle-foot orthosis, biological realistic leg prosthesis and forearm prosthesis. Since humanoids robots can perform any tasks like a human being. By using tools, operate equipment and vehicles designed for the human form, humanoids could theoretically perform any task like a human being can, as long as they have the proper software.

Humanoid robots are used for entertainments also. Once such example is Ursula, a female robot which can sings, play music, dances, and speaks to her audiences at Universal Studios. Even in Disney attractions the uses of animators are employed, robots like human beings look, move, and speak in some of their theme park shows. These animators look so realistic that it is harder to distinguish them from a distance whether or not they are actually human. Even though such robots have a realistic look, they have no cognition or physical autonomy. Various humanoid robots featured in an independent documentary film called Plug \& Pray, which was released in 2010.Humanoid robots. With artificial intelligence algorithms, are useful for future dangerous and/or distant space exploration missions.

Our main objective is to completely implement a bipedal robot which responds to commands given by human users via a mobile application including the capturing of images. We are trying to implement a prototype model of a humanoid robot with minimalistic hardware in order to maintain stability and make it economically viable and hence extract maximum uses from this intelligible design.

So in this paper we discuss on the following sections. In the next section the survey of humanoid robots is been studied. This is followed by working, results and conclusion. 


\section{LITERATURE SURVEY}

In [1] Al-Jazari described a band made up of humanoid automata which performed more than fifty facial and body actions during each musical selection. He also created hand washing automata with automatic humanoid servants, and an elephant clock incorporating an automatic humanoid mahout striking a cymbal on the half-hour. In his programmable castle clock five musician automata was featured which automatically played music when moved by levers operated by a hidden camshaft attached to a water wheel. In [2] Jacques de Vaucanson developed a Flute Player, a life-size figure of a shepherd that could play twelve songs on the flute and The Tambourine Player that played a flute and a drum or tambourine. Three laws of robotics was formulated by Isaac Asimov and was coined as the word robotics. In [3] Turkey's first dynamically walking humanoid robot, SURALP, was developed by SabanciUniversity in conjunction with Tubitak. In [4] Researchers at Japan's National Institute of Advanced Industrial Science and Technology demonstrated their humanoid robot HRP-4C singing and dancing along with human dancers .In [5] the Advanced Robotics Department in Italian Institute of Technology released its first version of the Complianthu Manoid robot Co Man which was designed for robust dynamic walking and balancing in rough terrain.

Due to the growing interest in this field, we decided to build a basic model of a biped robot to show how a simplified robot can be built.

\section{WORKING}

The block diagram of a transceiver section is shown in figure 1 .

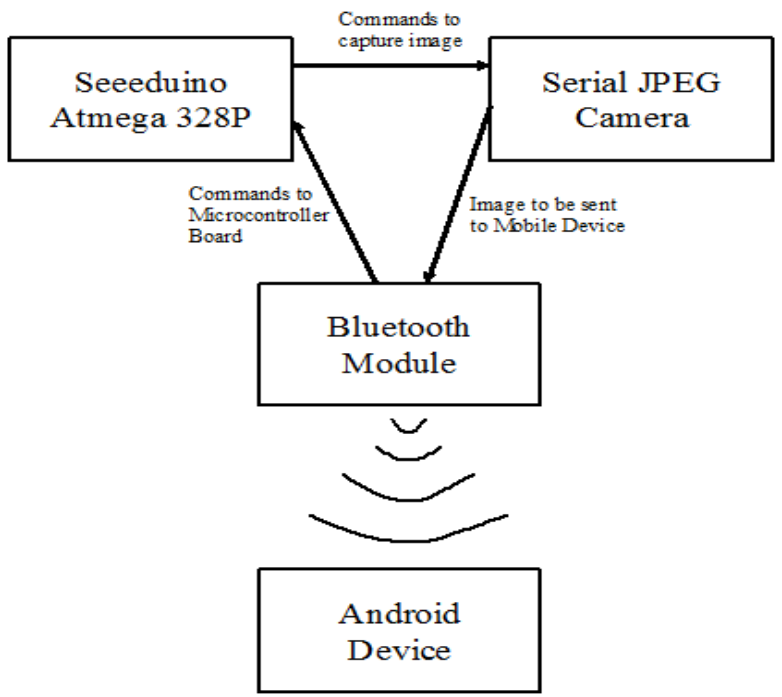

Figure 1. Transceiver section

The complete block diagram of the system is shown in figure 2. The ZENO module is shown in figure 6 . The working of the ZENO module is as follows.

\subsection{Working}

A coherent design of this model proves it to be easy to use. A servo motor is connected to the base plate of the robot's 'foot'. This motor is connected horizontally in order to move the foot sideways. The second motor is connected vertically near the 'hip' of the robot. The 2 motors on top of the robot are connected via a beam. To move the robot forward, the servo motor at the foot of the right leg is moved clockwise at a required angle. This causes the left leg of the robot to rise a little. Once this happens, the servo on the left hip is rotated clockwise while the one at the right hip is rotated anticlockwise to move the foot forward. Then the servo motor at the right foot is rotated anticlockwise to bring it back to its original position i.e., flat on the ground. Then, the left foot is moved to raise the right leg and move it forward similarly. The reverse of this process is done inorder to move the robot backwards. To turn left, a motion similar to the forward movement is carried out. However, only the left hip servo motor is rotated. The right hip servo motor remains constant. This process is mirrored for turning right. 


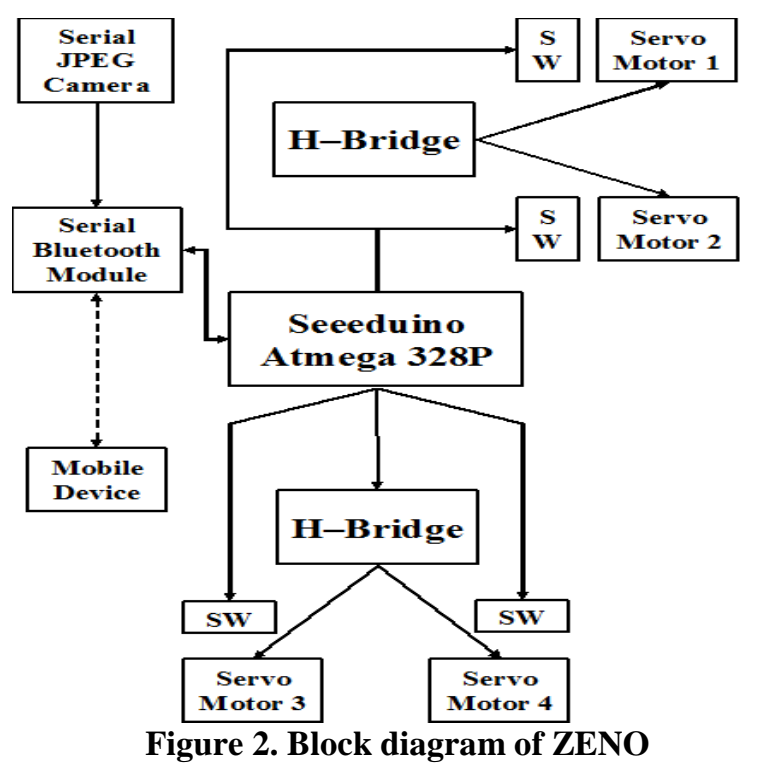

This entire movement is controlled using a mobile phone which has an application coded on Android Platform, which in turn is connected to each other via Bluetooth using a Bluetooth Module. The Android application links the Mobile Device to the Microcontroller Board via Bluetooth after taking the required permissions for binding. The application has the buttons displayed on the GUI for the sake of movement, namely: Forward, Backward, Left and Right. On pressing any of these, data is sent to the Bluetooth Module which in turn forwards the same data to the Microcontroller Board. Using these commands, the relevant section of the code is executed and thus resulting in the respective motion of the robot.

The robot has a Serial JPEG Camera that is based on TTL Logic which is attached to the Microcontroller Board. This is primarily used to capture images while the robot is in motion. The Mobile Application also has a button called "Capture Image" which on being pressed sends a signal to the Bluetooth Module which in turn sends the data to the Microcontroller Board. The Microcontroller Board then requests the Camera to capture an image and send the same to the Bluetooth Module which finally appears on the Mobile Screen. This can be used for several applications including surveillance, identification of structures, etc.

\subsection{Modular Description}

The component count in the project is a bare minimum as most of the required job is done by the highly versatile Microcontroller Board. The entire hardware can be split into blocks for the sake of understanding and the blocks are:

\section{a) Microcontroller Block:}

Seeeduino is an Arduino compatible board. It is based on the Duemilanove schematic, 100\% compatible to its existing program, shield and IDEs. On the hardware design, remarkable changes are taken to improve the flexibility and user experience. This consists of an ATMEGA328P microcontroller which has an operating voltage of $5 \mathrm{~V}$ or $3.3 \mathrm{~V}$.

The microcontroller is the central brain portion of this project. This component has the following various functions:

-It contains the walking code written using Arduino Coding Language stored in its memory

- It accepts data from the Bluetooth Module

- It sends commands to the Serial JPEG Camera

- It decodes the data sent from the Bluetooth Module and decides what action to take

- It executes the walking logic to suit the requirements of the user

\section{b) Bluetooth Module :}

This module is the main component of the wireless communication dependent portions of the robot. Bluetooth module offers an affordable way to let your microcontroller talk to your Bluetooth devices such as Bluetooth mobile phones, laptop, and Bluetooth adapter. The Bluetooth module provides TTL level UART interface which is supported by almost every microcontroller in the market. It is also designed to be compatible with most popular Arduino controller. Simply plug into Arduino IO Expansion Shield, a Bluetooth Arduino is ready to use. 


\section{c) Serial JPEG Camera Module:}

LS-Y201 is LinkSprite's new generation serial port camera module. It can capture high resolution pictures using the serial port. LS-Y201 is a modular design that outputs JPEG images through UART, and can be easily integrated into existing design. The serial JPEG Camera is being used to take images of the surroundings by Zeno as and when the user requires. The working of this module is primarily controlled by the microcontroller board. The code in the microcontroller board is used to accept data from Bluetooth module regarding button press and Request the camera to capture an image and send it to the mobile device.

The size of this module is $32 \mathrm{~mm} \times 32 \mathrm{~mm}$ with an image sensor consisting of a CMOS of size $1 / 4$ inches. The pixel size of this camera is 5.6 micrometers and the output format is standard JPEG. The camera is extremely sensitive and equipped with automatic exposure and gain and an electronic rolling shutter. The dynamic range is $60 \mathrm{~dB}$ with a maximum analog gain of $16 \mathrm{~dB}$ and the signal to noise ratio is $45 \mathrm{~dB}$. The monitoring distance of this is 10-15 meters maximum which is also adjustable in nature. The default baud rate is 38400 which is what we are using in the project.

The Arduino board initializes the camera and the Bluetooth module. Since the camera transmitter is fed to the Bluetooth receiver, the data is received. The module uses Serial Port Profile (SPP). The Serial Port Profile defines the requirements for Bluetooth devices necessary for setting up emulated serial cable connections using RFCOMM between two peer devices. The requirements are expressed in terms of services provided to applications, and by defining the features and procedures that are required for interoperability between Bluetooth devices.

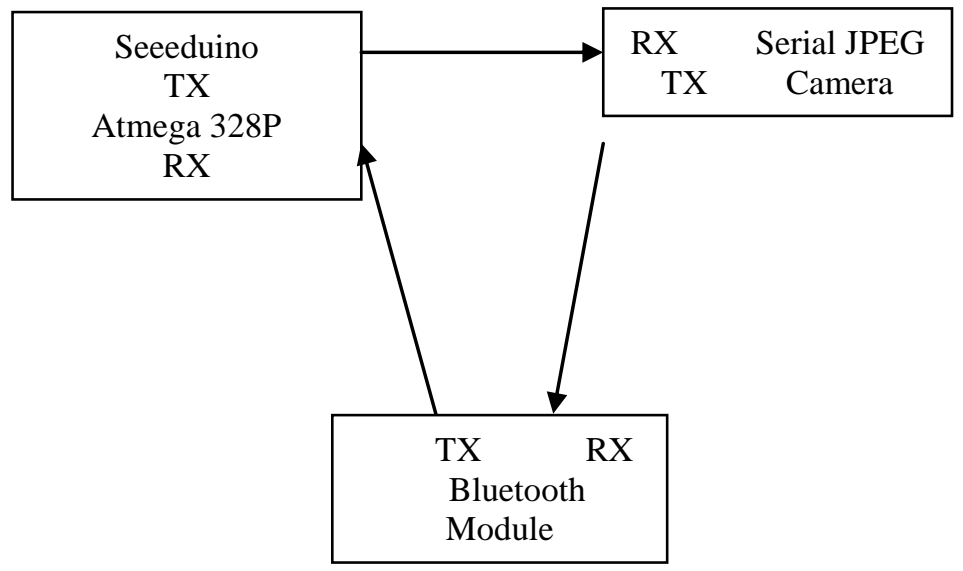

Figure 3. Connections between Microcontroller Board and Camera

The code in the microcontroller board is used to do the following things:

- Accept data from Bluetooth module regarding button press

- Request the camera to capture an image and send it to the mobile device.

- General Purpose Register File Hardware needed

1) The camera itself

2) $5 \mathrm{~V}$ power supply

3) RS232 Cable with DB9 female connector

- Communication Protocol

1. Reset

$\begin{array}{cc}\text { Command (HEX) } & \text { Return (HEX) } \\ 56002600 & 76002600\end{array}$

2. Take picture

Command (HEX) Return (HEX)

$560360100 \quad 7600360000$

3. Read JPEG file size

Command (HEX)

Return (HEX)

5600340100

76003400040000 XH XL 
$\mathrm{XH}$ XL is the file length of JPEG file, MSB is in the front, and followed by LSB.

\section{Read JPEG file content}

JPEG file starts with FF D8 and ends with FF D9. To read the JPEG file, always starts with address 00 00 , and choose a chunk size that are an integer times of 8 , and read the chunk many times until reds FF D9 which indicates the end of the JPEG file. The flowchart for initializing the JPEG Camera and reading a JPEG file is shown in figure 4 and 5.

\section{d) Servo Motors and Motor Drivers}

A servo motor consists of several main parts, the motor and gearbox, a position sensor, an error amplifier and motor driver and a circuit to decode the requested position. An $\mathrm{H}$ bridge is an electronic circuit that enables a voltage to be applied across a load in either direction. These circuits are often used in robotics and other applications to allow DC motors to run forwards and backwards. $\mathrm{H}$ bridges are available as integrated circuits or can be built from discrete components. In this project we are using two H-Bridges because each H-Bridge controls two motors. Since there are 4 motors being used here, we need two H-bridges. These devices also help us control the power being given to the driving motors also. Thus incidents of unexpected power surges harming the servo motors can be minimized. The method of control of these boards is via the Seeeduino board

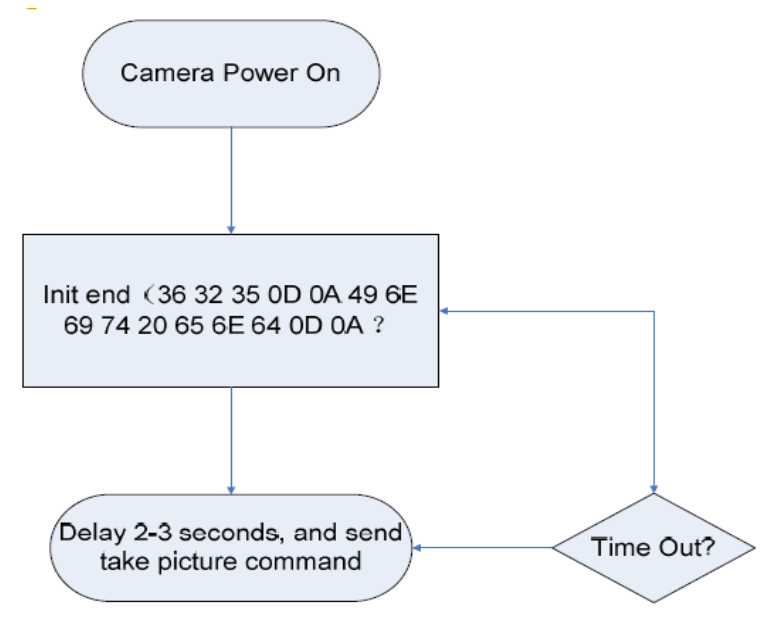

Figure 4. Initialization of JPEG Camera

The software coding is done using two platforms, namely Arduino and Android.

\section{RESULTS}

Zeno is successfully implemented and can be rightly termed cognizant. A large number of applications and advantages thus emerge from this implementation. We have designed a low cost prototype bipedal robot that responds to commands input by a human user. The stability has also been increased by using minimal and economic components and the resulting prototype can be used for any type of surveillance purposes. The prototype simulates human gait approximately and can be made available to the general public through the intelligible design.

The microcontroller board on ZENO is shown in Figure 7.The Bluetooth module and H-Bridge are shown in Figure 8 and 9. The application layout on Android device for the start up is shown in figure 10. The screenshot of the application after capturing the image is shown in figure 11. 


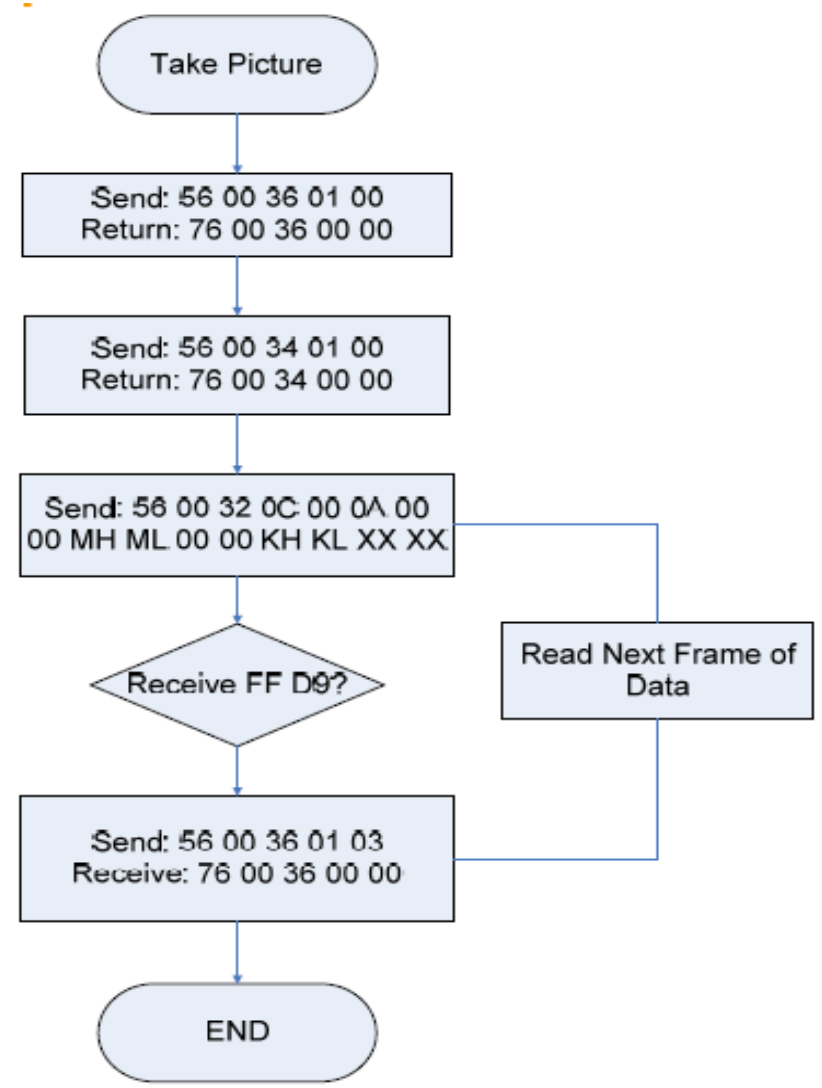

Figure5. Reading a JPEG File

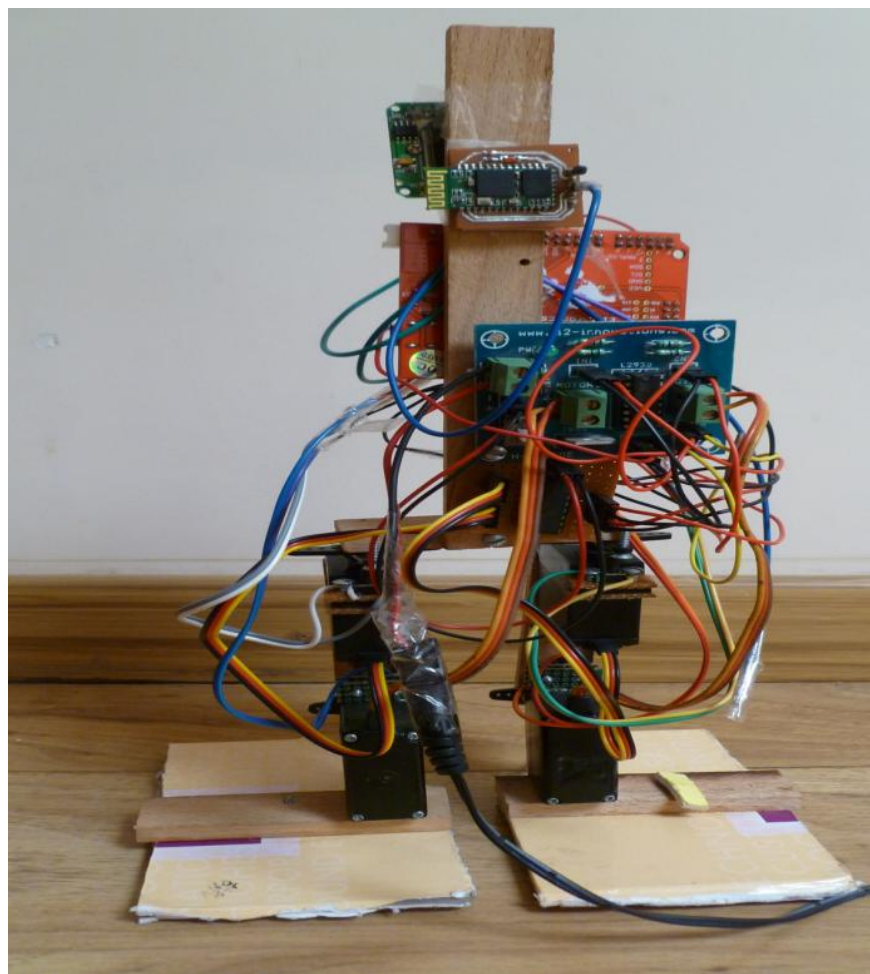

Figure 6. Module of ZENO 


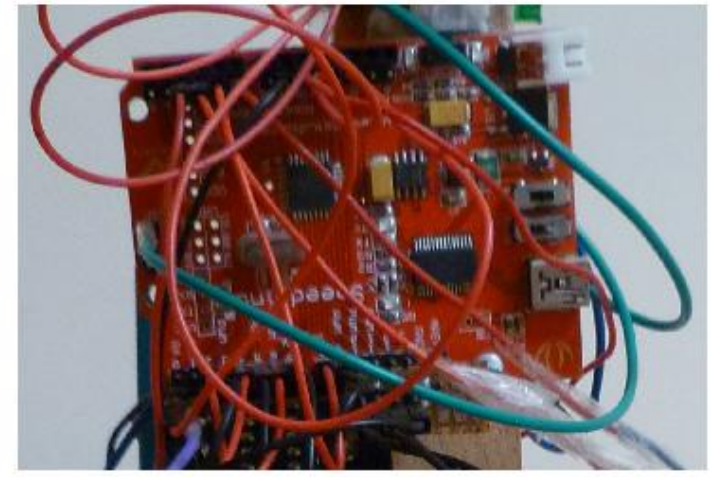

Figure 7. The microcontroller Board on ZENO

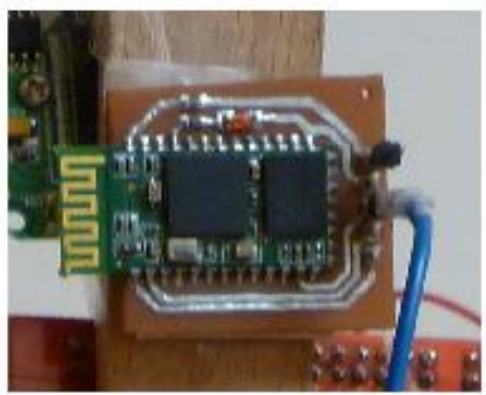

Figure 8. Bluetooth Module on ZENO

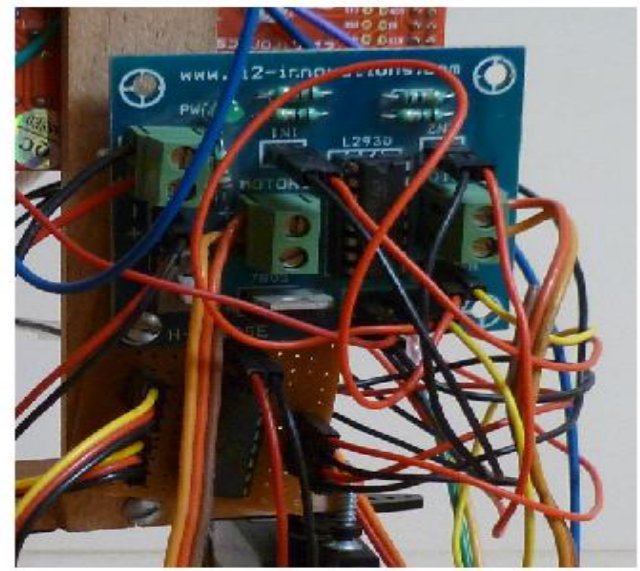

Figure 9. H-Bridge on ZENO 


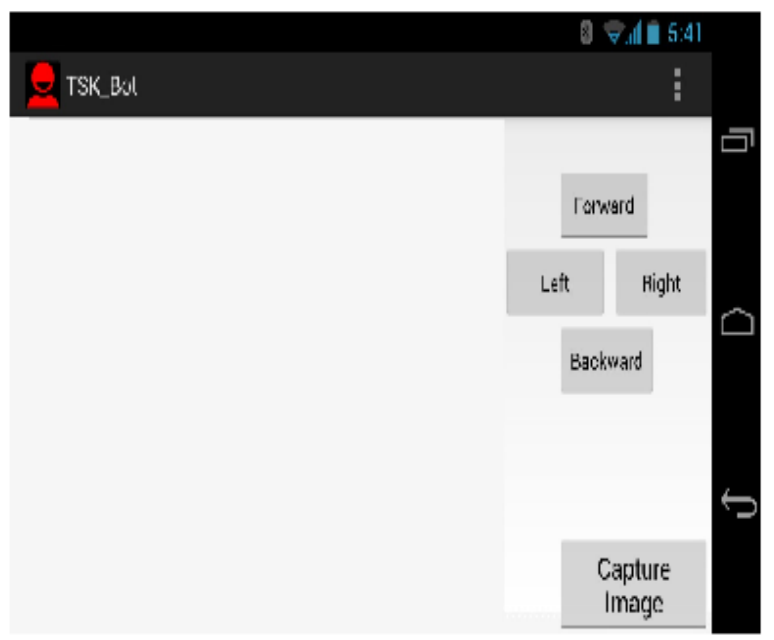

Figure 10. Application Layout on Android Devices for start up

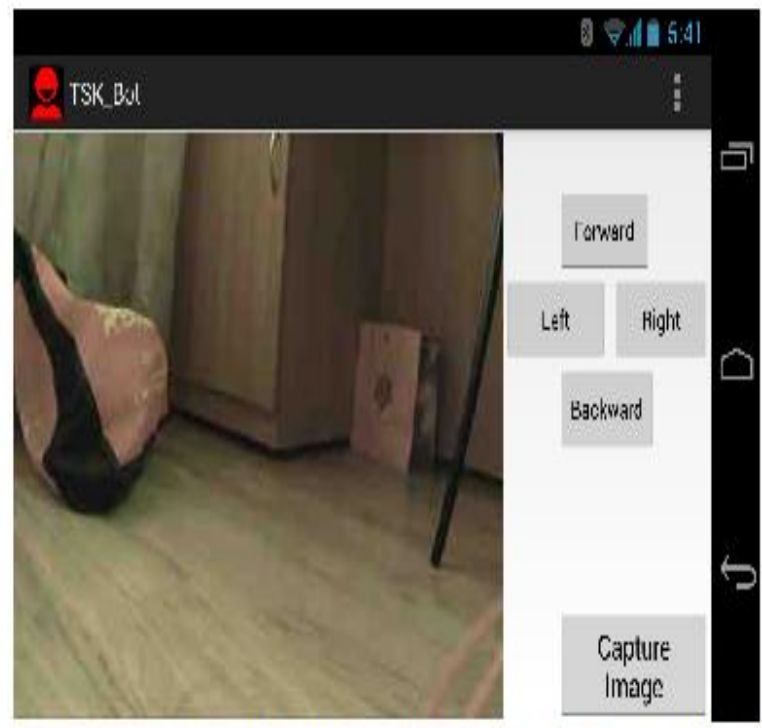

Figure 11. Screen shot of the application after capturing an image

\section{CONCLUSION}

This work reports the development of a bipedal robot. This robot has features of small size and light weight. The light weight property could help to achieve a faster speed at walk. The robot's design includes the incorporation of a camera for providing vision to the biped. A program to simulate the robot's walking was used. In order to improve the stability of the bipedal robot some modifications could be implemented as future work. The robot could be redesigned reducing the leg's mass in proportion to the trunk's mass. A problem in this modification is a possible servos overload due to an excessive mass addition to the trunk. A second modification is to increase the degrees of freedom at the hip, allowing the body to have lateral movements to facilitate control.

\section{REFERENCES}

[1] Rosheim, Mark E. (1994). Robot Evolution: The Development of Anthrobotics. Wiley-IEEE. pp. 9-10. ISBN 0-471-02622-0

[2] Resolved motion rate control of manipulators and human prostheses DE Whitney - IEEE Transactions on Man-Machine Systems, 1969

[3] Joseph Needham (1986), Science and Civilization in China: Volume 2, p. 53, England: CambridgeUniversity Press

[4] http://spectrum.ieee.org/automaton/robotics/humanoids/how-to-make-a-robot-dance

[5] http://www.iit.it/en/advr-labs/humanoids-a-human-centred-mechatronics/advr-humanoidsprojects/compliant-humanoid-platform-coman.html 